WIENER SLAVISTISCHES JAHRBUCH, Band 53/2007, 27-35

(C) 2007 by Österreichische Akademie der Wissenschaften, Wien

GEORG HOLZER

\title{
Zur Frage der Nordgrenze des slowenischen Sprachgebiets im Mittelalter
}

\begin{abstract}
V srednjem veku se je tudi v vzhodni Avstriji, med slovenskim in češkim govornim področjem, govorilo slovansko. Vendar so Slovani od Gorenjske do Nižje Avstrije živeli pod bavarsko oblastjo. Njihov jezik je bil zato pod močnim bavarskim vplivom in sicer tako na severu, kjer so izumrli, kot tudi na še slovensko govorečem jugu. Če opredelimo slovenščino kot slovanščino $\mathrm{z}$ bavarskim superstratom (kajti to jo razlikuje od vseh drugih slovanskih jezikov), potem se je njeno govorno področje v srednjem veku raztezalo vse tja do severne Nižje Avstrije.
\end{abstract}

Die Frage der Nordgrenze des slowenischen Sprachgebiets in der heutigen Zeit lässt sich verhältnismäßig klar beantworten. Man weiß oder könnte leicht feststellen, bis zu welcher Linie autochthone Slowenischsprachige leben. Auch wenn das slowenische und das deutsche Sprachgebiet einander überlappen, so hat doch jedes für sich seine Grenze. Im Mittelalter hingegen war die Situation eine ganz andere: Im Osten Österreichs, zwischen dem heutigen slowenischen und dem heutigen tschechischen Sprachgebiet, wurde damals ebenfalls Slavisch gesprochen, zunächst mehr oder weniger ausschließlich und ab dem 8. Jahrhundert zusammen mit dem Deutschen, das dann letzten Endes das Slavische verdrängte. Da aber stellt sich die Frage der Zugehörigkeit dieses mittlerweile verdrängten, d. h. ausgestorbenen Slavischen Ostösterreichs, nämlich ob es sich um das Slowenische handelte oder aber um das Tschechische oder ob es geteilt war: im Süden slowenisch und im Norden tschechisch, oder ob es gar eine eigene slavische Sprache war. Davon, welche dieser Möglichkeiten zutrifft, hängt ab, wo die Nordgrenze des slowenischen Sprachgebiets verlief.

In meinen Überlegungen zu dieser Frage werde ich mich auf hier mit 1. bis 9. bezifferte Thesen stützen, die ich großteils in früheren Arbeiten ausführlich begründet habe. Sie lauten:

1. Das Slavische zur Zeit der großen Expansion des Slaventums, also um ca. 600 n. Chr., war weitestgehend einheitlich. 
2. Alle Unterschiede zwischen slavischen Sprachen und Dialekten sind erst nach der Expansion, also nach 600 n. Chr. entstanden.

3. Demnach sind auch die slavischen Sprachen und Dialekte selber erst nach 600 n. Chr. entstanden, und zwar eben dort, wo sie dann historisch in Erscheinung treten.

4. Wie aus 1.-3. folgt, war das Slavische um 600 n. Chr. das Urslavische, von dem alle slavischen Sprachen und Dialekte abstammen.

5. Wie aus 1.-4. folgt, wurde Urslavisch auf dem gesamten Expansionsgebiet von 600 n. Chr. gesprochen.

6. Wie aus 1.-5. folgt, wurde nicht etwa ein bereits individualisiertes Slowenisch von irgendwoher in die heute slowenischen Landschaften verpflanzt, sondern das Slowenische entwickelte sich dort aus dem Urslavischen, wo es dann auch historisch greifbar wird, also in Krain und den anderen slowenischen Ländern. Die slavischen Einwanderer brachten das Urslavische, nicht das Slowenische nach Slowenien. ${ }^{1}$

7. Das Urslavische ist dadurch in verschiedene Dialekte zerfallen, dass an verschiedenen Stellen des urslavischen Sprachgebiets verschiedener Sprachwandel stattgefunden hat. Die von den verschiedenen Sprachwandeln erfassten Areale überlappten einander, was bedeutet, dass jeder slavische Dialekt ein Übergangsdialekt zwischen ihm benachbarten, einander aber gegenüberliegenden Dialekten war. Es handelte sich um das gemeinslavische Dialektkontinuum. ${ }^{2}$

Gut veranschaulichen lassen sich die in 7. dargestellten Verhältnisse anhand des Gailtaler Dialekts des Slowenischen. Dieser Dialekt hat sowohl, wie zu erwarten, mit seinen slowenischen Nachbardialekten gemeinsame Merkmale, als auch, wie weniger zu erwarten, wenngleich gut bekannt, mit dem Tschechischen, das im gemeinslavischen Dialektkontinuum ein mittelbarer Nachbar des Gailtaler Slowenischen war. Ein mit dem Tschechischen gemeinsames Merkmal ist die erhaltene urslavische Sequenz $d l$, die schon im südlich benachbarten Kanaltal ebenso wie im Krainer Slowenischen zu $l$ vereinfacht wurde ${ }^{3}$. Im Gailtal heißt zum Beispiel die 'Ahle' šidlo o. ä. ${ }^{4}$, jedenfalls mit erhaltenem $d$ wie im Tschechischen, während etwa das Krainer Slowenisch šilo hat.

Ein anderes Beispiel dieser Art bietet der längst ausgestorbene slavische Dialekt des niederösterreichischen Erlaftals. Dort sind urkundlich der Bergname Colomezza 'Pfahlgrenze' und der Personenname Leublinic 'Liebling' belegt. Colomezza reflektiert auf tschechische Art ursl. $d j$ durch $z$ (geschrieben $z z$ ) - slowenisch wäre es *Kolomeja -, und Leublinic enthält auf slowenische Art das epenthetische $l$ (vgl.

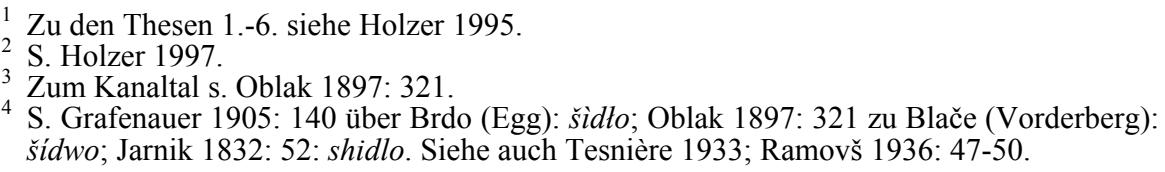


slowen. ljubljenik 'Liebling' gegenüber dem alttschechischen Personennamen $L$ ' $u$ $b$ 'en ohne epenthetisches $l$ ). ${ }^{5}$ Das Erlaftal liegt also in einem Bereich, in dem die 'Grenze' meza wie im Alttschechischen und nicht meja wie im Slowenischen hieß, in dem man dafür aber umgekehrt für 'geliebt' ljubljen wie im Slowenischen und nicht l'ub'en wie im Alttschechischen sagte.

Im Sinne der Thesen 1.-7. lassen sich diese beiden Beispiele auf folgende Weise in ein Gesamtbild einordnen: In urslavischer Zeit wurde auf dem gesamten slavischen Sprachgebiet *sj $\underline{\bar{u}}$ 'dla 'Ahle', *medj $\underline{\bar{a}}$ ' 'Grenze' und *ljawbjenu 'geliebt' gesagt, ${ }^{6}$ doch haben die verschiedenen Lautwandel ein Kontinuum hervorgebracht, wie es in untenstehender Tabelle dargestellt ist (die nachurslavischen Beispiele sind in einer idealisierten vorschriftlichen Lautung, aber ohne Asteriskus angeschrieben):

\begin{tabular}{llll} 
Ursl. & $*_{\text {sj } \underline{\underline{u}} \cdot d l a}$ & $*_{\text {medj } \underline{\bar{a}}} \cdot$ & $*$ ljawbjenu $>$ \\
\hline Böhmen: & šidlo & meza & ljubjen \\
Erlaftal: & šidlo & meza & ljubljen \\
Gailtal: & sidlo & meja & ljubljen \\
Kanaltal & šilo & meja & ljubljen
\end{tabular}

Tabelle: Ausschnitt aus dem gemeinslavischen Dialektkontinuum

Man sieht, es handelt sich um ein echtes Dialektkontinuum und um einen Ausschnitt aus dem gemeinslavischen Dialektkontinuum. Von Landschaft zu Landschaft ändert sich von den hier betrachteten Merkmalen immer nur eines: $d l z b j-d l z b l j-$ $d l j b l j-l j b l j$. Das mittelalterliche Slavische im Osten des heutigen Österreichs war je nördlicher, desto tschechischer und je südlicher, desto slowenischer. Eine Grenze zwischen dem Slowenischen und dem Tschechischen zeichnet sich da aus dialektgeographischer Sicht nicht ab.

Ein anderer Ansatz wäre, nicht die dialektgeographische, sondern die genetische Zugehörigkeit einer slavischen Mundart etwa in Niederösterreich festzustellen, also zu bestimmen, ob die Mundart sozusagen stammbaummäßig dem Slowenischen oder dem Tschechischen angehört. In dieser Überlegung ist von der 8 . These auszugehen:

5 S. Holzer 2001: 51-53 und $70 \mathrm{f}$.

${ }^{6}$ Der hochgestellte Punkt ${ }^{\circ}$ bezeichnet den Akut, die Unterstreichung die Betonung, s. Holzer 2005 und 2007b. Zur Rekonstruktion urslavischer Lautungen s. Holzer 1998, 2003d, $2005,2007 b$.

7 Für das Erlaftal ist šidlo zwar nicht belegt, kann aber aus dialektgeographischen Gründen in dieser Lautung angesetzt werden, nämlich weil beide Nachbarn, sowohl der nördliche als auch der südliche, in der Erhaltung des $d$ miteinander übereinstimmen. 
8. Sprachen (Dialekte mitverstanden) sind dann und nur dann miteinander genetisch verwandt, wenn es einmal eine Zeit gegeben hat, in der sie identisch, also nur eine einzige Sprache waren. Diese einzige Sprache ist die Ursprache der verwandten Sprachen. Verwandte Sprachen sind definitionsgemäß verschiedene Modifikationen einer einzigen Sprache, ihrer Ursprache. Sie bilden eine Familie von Sprachen. ${ }^{8}$

Dass die slowenischen Dialekte Mitglieder einer Sprachenfamilie sind, nämlich der slavischen, ist nicht zu bestreiten. Zu fragen bleibt aber, ob die heutigen slowenischen Dialekte alle miteinander näher verwandt sind, als es einzelne von ihnen mit anderen slavischen Dialekten, etwa mit tschechischen oder kroatischen sind. Mit anderen Worten, ob es einmal eine Zeit gegeben hat, in der alle heutigen slowenischen Dialekte noch miteinander identisch, aber bereits verschieden von den slavischen Dialekten der Umgebung waren. Dies hängt davon ab, ob der älteste Unterschied, der alle slowenischen Dialekte gemeinsam von allen nichtslowenischen Dialekten trennt, älter oder jünger ist als der älteste Unterschied, der beliebige zwei slowenische Dialekte voneinander trennt. Nur wenn er älter ist, hat es ein Urslowenisch gegeben und kann ein ausgestorbener slavischer Dialekt in Ostösterreich daraufhin untersucht werden, ob er vom (zu rekonstruierenden) Urslowenischen ableitbar ist, also genetisch gesehen ein slowenischer Dialekt war. Doch fehlt uns das Wissen, um hier eine Entscheidung zu treffen. Wir wissen ja weder, welcher der älteste Unterschied ist, der alle slowenischen Dialekte gemeinsam von allen nichtslowenischen Dialekten trennt, noch, welcher der älteste Unterschied ist, der beliebige zwei slowenische Dialekte voneinander trennt, geschweige denn wissen wir, welcher dieser beiden ältesten Unterschiede der ältere ist. So ist es eben unmöglich festzustellen, ob das Slowenische eine genetische Größe, also eine Dialektfamilie ist, und daher ist es auch müßig zu fragen, ob ein ausgestorbener slavischer Dialekt in Österreich in genetischem Sinne slowenisch war oder nicht. Die Frage nach der Nordgrenze des slowenischen Sprachgebiets im Mittelalter lässt sich also auch so nicht beantworten. ${ }^{9}$

Zum Ziel könnte aber ein sozio- und kontaktlinguistischer Zugang führen. Den Ausgangspunkt bildet die These 9 .

9. Altslowenisch ist das Slavisch des bairischen Ostlandes.

Das muss in einem kleinen historischen Exkurs näher erläutert werden. Im frühmittelalterlichen „bairischen Ostland“, das sich zwischen Donau, Traun und Drau erstreckte und auch das obere Savetal sowie bald auch das nördlich der Donau gelegene Waldviertel miteinschloss, also ganz grob gesprochen Niederösterreich, die

${ }_{9}^{8}$ Siehe Holzer 1996a: 13-48.

9 Vgl. die parallele Argumentation in Holzer 2002a: 1085 f. und 2007b: 20-23. Siehe auch 1996a: 13-48. 
Steiermark, Kärnten, Osttirol, Krain und das ungarische Transdanubien (westlich der Donau) umfasste, war die slavische Bevölkerung von einer bairischen überschichtet worden und haben die Sprachen der beiden Völker aufeinander eingewirkt. Historische Quellen belegen das slavisch-bairische Zusammenleben in diesen Gebieten. So berichtet die Conversio, das von Karl dem Großen eroberte Land coeperunt populi sive Sclavi vel Bagoarii inhabitare. In der Noticia bonorum de Lonka von 1160, dem Urbar der Güter des Bischofs von Freising in Škofja Loka, zu Deutsch Lack, heißt es: Bauuari possident nonaginta quatuor hobas [...] De Sclauis centum quinquaginta tres hobe [...] (hoba = Hube). In einer Urkunde von 976/979 ist von de Bauuaria missis colonis im Erlaftal die Rede und wird gleichzeitig die slavische Sprache erwähnt, indem es heißt: usque in montem, qui dicitur Sclauanice Rûznic. ${ }^{10}$ Und zum Waldviertel in Niederösterreich nördlich der Donau gibt es eine Urkunde von 902 oder 903, in der ein slavischer Adeliger dem Bischof von Freising Land und Leute in Stiefern am Kamp schenkt. Die Leute haben teilweise slavische Namen, so wie auch der Name Stiefern slavischen Ursprungs sein dürfte und eine Reihe anderer geographischer Namen im Kamptal sicher slavischer Herkunft sind. ${ }^{11}$

Unter diesen Umständen ist zu erwarten, und es ist auch belegbar, dass das Slavische im bairischen Ostland unter starker Einwirkung des Bairischen stand. Dies zeigen nicht nur bestimmte Eigenschaften des modernen Slowenischen, sondern auch des mittelalterlichen Slavischen im bairischen Ostland. Insbesondere ist hier an den slavischen Wortschatz der karolingischen Mission, des Rechts-, des Grundherrschafts-, des Militär- und des Handelswesens zu denken. Zwischen Niederösterreich und Krain gab es da kaum wesentliche Unterschiede. Die entstanden erst später, als Ostösterreich immer mehr germanisiert wurde, bis auf den letzten Rest in Kärnten, wo aber dieser Prozess weiter voranschreitet, in ununterbrochener Kontinuität seit dem Mittelalter. Man kann sagen, dass im Mittelalter die $\mathrm{R}$ a h m e n b e d i n g u n g e n d e s L e b e n s bei allen Slaven von Krain bis ins Waldviertel sehr ähnlich waren und alle diese Slaven zu einer Schicksalsgemeinschaft verbanden. Da es aber gemeinsame Rahmenbedingungen des Lebens mit sich bringen, dass in der betreffenden Gemeinschaft auch bestimmte terminologische Regelungen allgemeine Gültigkeit haben und bestimmte Texte, vor allem religiöse, von allen gekannt werden und stilistische Vorbildwirkung ausüben, bestimmen sie auf gewissen Ebenen, insbesondere auf der lexikalischen, semantischen, idiomatischen und stilistischen, den Sprachgebrauch der betreffenden Schicksalsgemeinschaft und schaffen Isoglossen, die das Siedlungsgebiet dieser Schicksalsgemeinschaft einschließen. ${ }^{12}$ Es bietet sich nun an, eben diese Isoglossen als maßgeblich anzusehen, wenn es gilt, ein Sprachgebiet zu definieren. Gegenüber lauthistorischen Isoglossen etwa haben sie zwei Vorzüge: Sie treten gebündelt und nicht einzeln gestreut auf und stellen somit

${ }^{10} \mathrm{Zu}$ den Quellen s. Holzer 2001: 24, 38 f., 2003b: $38 \mathrm{f}$.

11 S. Holzer 2003a und Hundsbichler 2003.

12 S. Holzer 2003c, 2006a, 2007a. 
deutliche Grenzen dar, und sie decken sich darüber hinaus mit extralinguistischen, nämlich die Rahmenbedingungen des Lebens betreffenden Grenzen, die von Sprachgemeinschaften oft als identitätsstiftend empfunden werden.

Sieht man es so, dann hätten die mittelalterlichen Slaven von Krain bis ins Waldviertel dieselbe Sprache bzw. Dialekte derselben Sprache gesprochen. Diese Sprache, das Slavische des bairischen Ostlandes, kann man im Hinblick darauf, dass das moderne Slowenisch ihren letzten und einzigen Rest darstellt, Altslowenisch nennen. Die Nordgrenze des so definierten Altslowenischen wäre dann nördlich des Kamptales in Niederösterreich verlaufen.

Diese Sichtweise hat auch für die sprachliche Einordnung der $\mathrm{F} \mathrm{r}$ e i s i n g e r $\mathrm{D}$ e $\mathrm{n}$ k m ä 1 e $\mathrm{r}$ ihre Relevanz. Die Freisinger Denkmäler repräsentieren ein slavisches Idiom, von dem es heute keinen Nachkommen mehr gibt. Es hat modliti 'beten' mit der erhaltenen Sequenz $d l$, wie sie auch im Gailtalslowenischen und im Tschechischen erhalten ist, steht aber dem Tschechischen noch näher als das Gailtalslowenische, z. B. weil es vueru 'den Glauben' hat wie tschechisch věru und nicht wie slowenisch vero. Andererseits hat es südslavisches razumeti, wogegen es im Tschechischen rozuméti 'verstehen' heißt. Aus dialektgeographischen Gründen ist klar, dass die Sprache der Freisinger Denkmäler nicht aus einer Landschaft stammt, in der heute noch Slowenisch gesprochen wird, sondern aus einem weiter nördlich, dem Tschechischen näher gelegenen Gebiet, wohl aus einem, das heute in Österreich liegt. Sind sie dann überhaupt slowenisch? Definiert man das Altslowenische als das Slavische des bairischen Ostlandes, dann kann man die Sprache der Freisinger Denkmäler vorbehaltlos als Altslowenisch betrachten. Auch lassen die Texte der Freisinger Denkmäler den bairischen Einfluss erkennen, und zwar in den Lehnprägungen, in den Anleihen am altbairischen St. Emmeram-Gebet und in der bairischalthochdeutschen Orthographie. Auch dass die Freisinger Denkmäler in das Pontifikale des Bischofs von Freising - übrigens demselben Freising, dessen Bischöfe Grundherren in Lack und am Kamp waren - eingebunden gefunden worden sind, zeigt die Abhängigkeit dieses Denkmals vom mittelalterlichen Baierntum. ${ }^{13}$ All das - aber auch n u r das - macht die Freisinger Denkmäler zu einem Dokument der mittelalterlichen slowenischen Sprache. ${ }^{14}$

${ }^{13}$ Zu den Freisinger Denkmälern Holzer 1986, 1996b: 102 f., 2003c: 219-221 (mit Literaturhinweisen), Bergermayer 2006: $7 \mathrm{ff}$.

14 Denkt man hier konsequent weiter, müssten auch die Kiever Blätter in die Betrachtungen miteinbezogen werden. Sie stammen wahrscheinlich aus dem slavischen Fürstentum am Plattensee, also einem Gebiet, das bis zum Ungarnsturm um die Wende vom 9. zum 10. Jahrhundert ebenfalls zum bairischen Ostland gehörte. Falls der Text der Kiever Blätter vor dem Ungarnsturm entstanden ist, wäre er in demselben Sinn wie die Freisinger Denkmäler als altslowenisch zu betrachten, auch wenn seine Sprache dialektgeographisch vom heutigen Slowenischen bedeutend mehr Abstand hat als die Sprache der Freisinger Denkmäler. Zu den Kiever Blättern s. die Literaturangaben in Holzer 1996b: 84, Fußnote 4 sowie Holzer 2003c: 221 (mit Literaturhinweisen). 
Auch zu Karantanien lässt sich aus diesem Blickwinkel etwas sagen. Nach Herwig Wolfram gehörten nur etwa 10-15\% des heutigen slowenischen Staatsgebietes zu Karantanien, ${ }^{15}$ welches vielmehr fast zur Gänze innerhalb der heutigen Grenzen Österreichs liegt. ${ }^{16}$ Karantanien und Slowenien sind einander eher benachbart als deckungsgleich. War das Slavische Karantaniens dann überhaupt slowenisch? Insofern ja, als Karantanien Teil des bairischen Ostlandes war.

Wenn man auf diese Weise über das mittelalterliche Slowenische nachdenkt, dann war sein Gebiet sehr groß und verlief seine Nordgrenze sehr weit im Norden. Diese gegenüber dem Slowenischen ziemlich großzügige Sicht der Dinge hat aber ihren Preis: Sie erfordert, dass man das Slowenische in Bezug auf eine andere Sprache, das Bairische, definiert. Denn nur dieser Bezug bildet die Klammer, die das Slavische Niederösterreichs, ja sogar das Slavische Karantaniens und das der Freisinger Denkmäler mit dem Slavischen Krains und der anderen modernen slowenischen Länder zusammenhält.

\section{Literatur}

Bergermayer 2006: Angela Bergermayer, Slavisches in den Namen von Untertanen des Bistums Freising im Mittelalter, Wiener Slavistisches Jahrbuch 52, 7 ff., im Druck

Daim - Szameit 1998: Falko Daim - Erik Szameit, Der Ostalpen- und Donauraum 788 (vor den Awarenkriegen), in: F. W. Putzger - E. Bruckmüller (Hg.), Historischer Weltatlas zur allgemeinen und österreichischen Geschichte. Neubearbeitung 1998, Wien, 21/I

Grafenauer 1905: $\quad$ Ivan Grafenauer, Zum Accente im Gailthalerdialekte, Archiv für slavische Philologie XXVII (1905) 195-228

Holzer 1986: $\quad$ Georg Holzer, Die Reflexe des hinteren Nasalvokals * $\mathrm{g}$ in den Freisinger Denkmälern, Wiener Slavistisches Jahrbuch 32, 29-35

Holzer 1995: $\quad$ Georg Holzer, Die Einheitlichkeit des Slavischen um 600 n. Chr. und ihr Zerfall, Wiener Slavistisches Jahrbuch 41, 55-89

Holzer 1996a: Georg Holzer, Das Erschließen unbelegter Sprachen. Zu den theoretischen Grundlagen der genetischen Linguistik (= Schriften über Sprachen und Texte 1), Frankfurt am Main u. a.

Holzer 1996b: $\quad$ Georg Holzer, Zu Lautgeschichte und Dialekten des mittelalterlichen Slavischen in Österreich, Wiener Slavistisches Jahrbuch 42, 81-110

Holzer 1997: $\quad$ Georg Holzer, Zum gemeinslavischen Dialektkontinuum, Wiener Slavistisches Jahrbuch 43, 87-102

Holzer 1998: $\quad$ Georg Holzer, Zur Rekonstruktion urslavischer Lautungen, in: Prasłowiańszczyzna i jej rozpad, hrsg. von Jerzy Rusek und Wiesław Boryś, Warszawa, 57-72

Holzer 2001: $\quad$ Georg Holzer, Die Slaven im Erlaftal. Eine Namenlandschaft in Niederösterreich (= Studien und Forschungen aus dem Niederösterreichi-

15 S. Wolfram 1995: 301.

16 Vgl. die Karten von Daim - Szameit 1998, von H. Dopsch bei Wolfram 1995: 258 und in Korošec 1986: 106. 
Holzer 2002a:

Holzer 2002b:

Holzer 2003a:

Holzer 2003b:

Holzer 2003c:

Holzer 2003d:

Holzer 2005:

Holzer 2006a:

Holzer 2007a:

Holzer 2007b:

Hundsbichler 2003:

Jarnik 1832:

Korošec 1986:

Oblak 1897:

Ramovš 1936: schen Institut für Landeskunde, hrsg. von Anton Eggendorfer und Willibald Rosner, Band 29 = NÖ Schriften 136 Wissenschaft), Wien Georg Holzer, Problemi vanjskih i unutrašnjih granica južnoslavenskoga jezičnog prostora, Forum, godište XXXXI, knjiga LXXIII, broj 7-9, Zagreb, jesen 2002, 1078-1091

Georg Holzer, Zur Sprache des mittelalterlichen Slaventums in Österreich. Slavisch unter bairischem Einfluss, Wiener Slavistisches Jahrbuch 48, 53-73

Georg Holzer, Der Name Stiefern, in: Helmut Hundsbichler (Red.), Andere Zeiten. Jubiläumsbuch 1100 Jahre Stiefern. Mit 555 Abbildungen, Stiefern, 92 f. Und: Georg Holzer, Zur slavischen Vergangenheit des Kamptales, im selben Band, 86

Georg Holzer, Slavisches Altertum im Erlaftal im Lichte von Namenkunde und Philologie, in: Regionalgeschichte am Beispiel von Scheibbs in Niederösterreich. Die Vorträge des 22. Symposions des Niederösterreichischen Instituts für Landeskunde Scheibbs, 1. bis 4. Juli 2002, hrsg. von Ursula Klingenböck und Martin Scheutz (= Studien und Forschungen aus dem Niederösterreichischen Institut für Landeskunde, hrsg. von Anton Eggendorfer und Willibald Rosner, Band $35=$ NÖ Schriften 147 Wissenschaft), St. Pölten, 25-52

Georg Holzer, Zum Wortschatz des slavischen Substrats in Österreich I. Schichten und Areale, in: Studia etymologica Brunensia 2, hrsg. von Ilona Janyšková und Helena Karlíková, Praha, 215-227

Georg Holzer, Urslavische Phonologie, Wiener Slavistisches Jahrbuch 49, $23-40$

Georg Holzer, Zur relativen Datierung prosodischer Prozesse im Gemeinslavischen und frühen Kroatischen, Wiener Slavistisches Jahrbuch 51, 31-71

Georg Holzer, Das bairische Ostland und seine Slaven, in: Studia Philologica Slavica. Festschrift für Gerhard Birkfellner zum 65. Geburtstag gewidmet von Freunden, Kollegen und Schülern. Teilband I, hrsg. von Bernhard Symanzik (= Münstersche Texte zur Slavistik 4), Berlin, 175-189

Georg Holzer, $\mathrm{Zu}$ den Slavica in der Kremsmünsterer Urkunde von 777, in: Wort - Geist - Kultur. Gedenkschrift für Sergej S. Averincev, hrsg. von Juliane Besters-Dilger, Heinz Miklas, Gerhard Neweklowsky und Fedor B. Poljakov (= Russkaja kul'tura v Evrope / Russian Culture in Europe 2), Frankfurt am Main u. a., 27-46

Georg Holzer, Historische Grammatik des Kroatischen. Einleitung und Lautgeschichte der Standardsprache (= Schriften über Sprachen und Texte 9), Frankfurt am Main u. a.

Helmut Hundsbichler, 902/903: Joseph, Freising, Stiuinna, in: Helmut Hundsbichler (Red.), Andere Zeiten. Jubiläumsbuch 1100 Jahre Stiefern. Mit 555 Abbildungen, Stiefern, 80 f. Und: Helmut Hundsbichler, Der Text der ersten Nennung 902 oder 903, im selben Band, $82 \mathrm{f}$. Und: Helmut Hundsbichler, Wer war Joseph?, im selben Band, $84 \mathrm{f}$. Und: Helmut Hundsbichler, Die 902 oder 903 übergebenen Gebiete, im selben Band, $90 \mathrm{f}$.

Urban Jarnik, Versuch eines Etymologikons der slowenischen Mundart in Inner-Österreich, Klagenfurt

Paola Korošec, Alpenslawen und Slowenen, in: Welt der Slawen. Geschichte, Gesellschaft, Kultur, hrsg. von Joachim Herrmann, München, 103-108

Vatroslav Oblak, Kleine grammatische Beiträge, Archiv für slavische Philologie XIX, 321-328

Fran Ramovš, Kratka zgodovina slovenskega jezika I, Ljubljana 1936, Nachdruck 1995 
Tesnière 1933: $\quad$ Lucien Tesnière, Les diphones tl, dl en slave: essai de géolinguistique, Revue des Études slaves XIII, 51-100

Wolfram 1995: Herwig Wolfram, Grenzen und Räume. Geschichte Österreichs vor seiner Entstehung (Österreichische Geschichte 378-907, hrsg. von Herwig Wolfram), Wien

Georg Holzer

Institut für Slawistik der Universität Wien

Universitätscampus AAKH, Hof 3

Spitalgasse 2, A-1090 Wien

georg.holzer@univie.ac.at 
